\title{
Diseño y construcción de un acoplamiento electrónico para realizar conexiones de IoT en un PLC convencional
}

\author{
Design and construction of an electronic coupling to make IoT connections in a \\ conventional PLC
}

CAMACHO-ALTAMIRANO, Ulices $\dagger^{*}$, MARTÍNEZ-CARRILLO, Irma y JUÁREZ-TOLEDO, Carlos

Universidad Autónoma del Estado de México

ID $1^{\text {er }}$ Autor: Ulices, Camacho-Altamirano / ORC ID: 0000-0002-4902-6936, Researcher ID Thomson: G-1804-2018, CVU CONACYT ID: 784595

ID $1^{\text {er }}$ Coautor: Irma, Martínez-Carrillo / ORC ID -0000-0002-7952-4418, Researcher ID Thomson: B-9264-2016 CVU CONACYT ID: 39914

ID $2^{\text {do }}$ Coautor: Carlos, Juárez-Toledo / ORC ID -0000-0002-7440-3246, Researcher ID Thomson, C-1368-2016, CVU CONACYT ID: 39912

DOI: $10.35429 /$ JCA.2019.10.3.8.14

Recibido Abril 30, 2019; Aceptado Junio 30, 2019

\begin{abstract}
Resumen
El crecimiento de la tecnología digital industrial (Industria 4.0) es un proceso que permite recopilar, controlar y analizar datos sobre varios procedimientos técnicos. El resultado de la aplicación de esta tecnología es la obtención de productos de mayor calidad a un menor costo. Cada vez es más difícil ignorar el papel que tiene el Internet de las Cosas (IoT) en los procesos productivos, sin embargo, esta tecnología es costosa y requiere de una actualización de los Controladores Lógicos Programables (PLCs). Este artículo se centra en el diseño y la construcción de un dispositivo de acoplamiento electrónico para realizar conexiones de IoT en un PLC convencional. Se utiliza un PLC Siemens S7-200, donde a través de las entradas y salidas de este dispositivo se acopla a una tarjeta nodemcu esp32 WIFI para monitorear y controlar el proceso. Se incluyen el circuito electrónico y el diagrama de flujo del acoplamiento entre el PLC y la tarjeta WIFI. La evidencia de este estudio sugiere que la actualización de los equipos industriales hacia la industria 4.0 es posible sin la necesidad de realizar cambios significativos.
\end{abstract}

Industria 4.0, Internet de las Cosas, Controladores Lógicos Programables

\begin{abstract}
The growth of the digital industrial technology (Industry 4.0 ) is a revolution that makes it possible to gather, control and analyze data about several technical procedures. The result of this manufacturing revolution is the higherquality products being produced at a lower cost. It is becoming increasingly difficult to ignore the role that Internet of Things (IoT) has in the productive processes, however, this technology is expensive and requires an update of the Programmable Logic Controllers (PLCs). This paper will focus on the update of the design and construction of an electronic coupling device to make IoT connections in a conventional PLC. In this work a siemens S7-200 PLC is used, in the inputs and outputs of this device a nodemcu esp32 WIFI card is coupled to monitor and control the process. The electronic circuit and the flow diagram of the coupling between the PLC and WIFI card are included. The evidence from this study suggests that the upgrade of the industrial equipment towards the industry 4.0 is possible without the need to make significant changes.
\end{abstract}

Industry 4.0, Internet Of Things, Programmable Logic Controllers

Citación: CAMACHO-ALTAMIRANO, Ulices, MARTÍNEZ-CARRILLO, Irma y JUÁREZ-TOLEDO, Carlos. Diseño y construcción de un acoplamiento electrónico para realizar conexiones de IoT en un PLC convencional. Revista de Cómputo Aplicado. 2019, 3-10: 8-14

\footnotetext{
* Correspondencia al Autor (Correo electrónico: ucamachoa713@alumno.uaemex.mx)

$\dagger$ Investigador contribuyendo como primer Autor.
} 


\section{Introducción}

A partir de principios del siglo XXI la cuarta Revolución Industrial también conocida como Revolución Digital o Industria 4.0 es generada por el efecto combinatorio de tecnología física, digital y biológica, donde las áreas de estudio con mayor interés son (Schwab 2016):

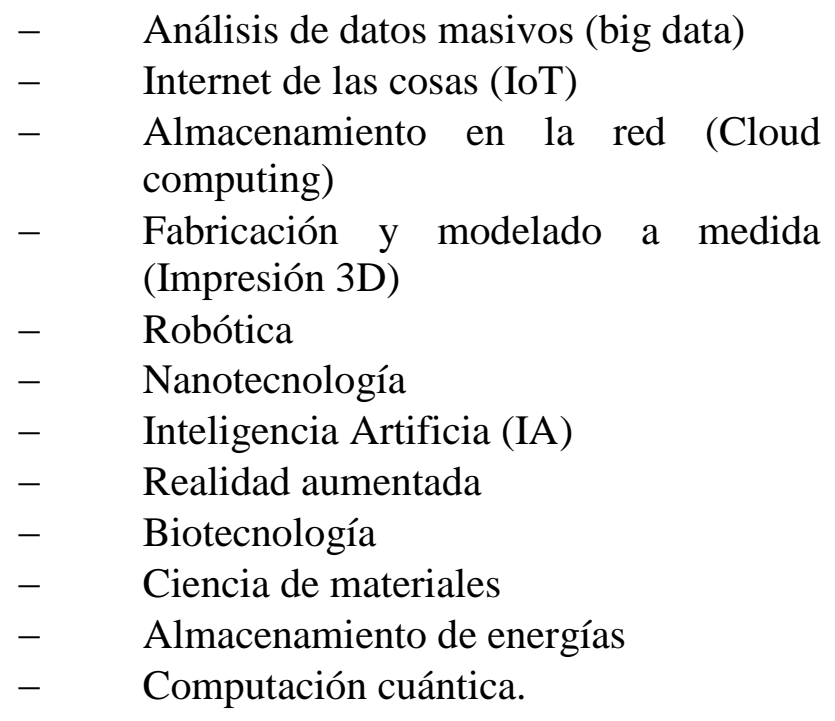

La interconexión digital entre objetos de uso cotidiano es lo que se conoce como internet de las cosas (IoT), esta comunicación permite aprender e interactuar a partir de dispositivos conectados a internet (Esfahani 2017), donde la World Wide Web (www) ha provocado una revolución en la comunicación entre las personas y los objetos, concediendo la integración del mundo cibernético con el mundo físico (Ngu 2016).

Minoly (2017), hace mención en la comunicación Máquina a Máquina, los vehículos autónomos (Cheng 2018), impresiones 3D, sensores más pequeños y eficientes (Muñiz 2019), (RFID) monitoreo por radiofrecuencia (Zhang 2018), sistemas de seguridad (Pancha 2019), ciudades inteligentes (Lau 2017), redes inteligentes, hogares inteligentes (Quispe y Maquera 2019), salud electrónica (Humayed 2017), gestión de activos y logística, agricultura (Ahmed 2018), control y monitoreo industrial (Tietz 2018) entre otros, son algunas aplicaciones mostradas en la figura 1 que corresponden al auge del internet de las cosas.

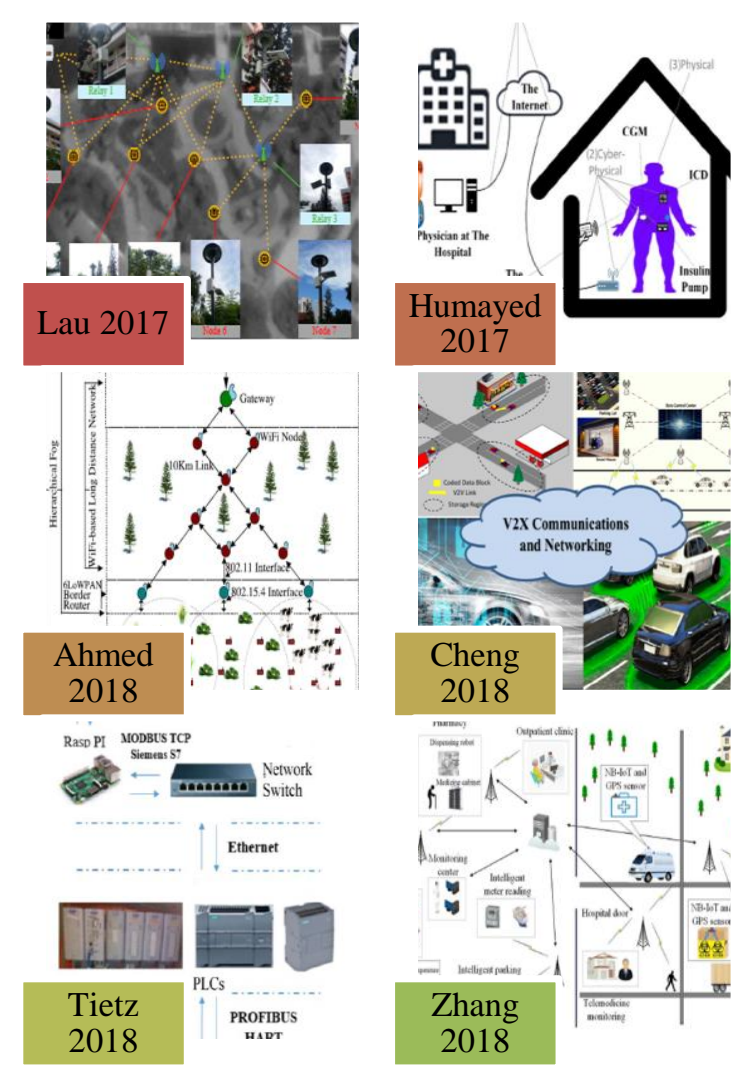

Figura 1 Aplicaciones de sistemas de IoT Fuente: Elaboración Propia

Uno de los principales problemas de comunicación entre microcontroladores y PLC comerciales ha sido a que convencionalmente trabajan en voltajes diferentes. En este trabajo se diseña e implementa un dispositivo de acoplamiento electrónico para realizar conexiones de IoT y un controlador lógico programables (PLC) en tiempo real con el fin de monitorear y controlar el proceso.

La obtención de la información se maneja mediante una tarjeta de adquisición de datos múltiples que ha sido interconectada con el PLC usando 24 VDC a 5 VDC y viceversa en el circuito electrónico. Pinto y Arias (2019) consideran que la finalidad del procesamiento de información en las tarjetas de adquisición de datos es tener la capacidad de realizar las funciones de control y monitoreo en redes eléctricas

De acuerdo con Quian (2017) las redes híbridas de comunicación resuelven el problema de monitoreo mediante sensores inalámbricos móviles y PLCs donde la comunicación entre ambos se realiza con el internet de las cosas (IoT). 


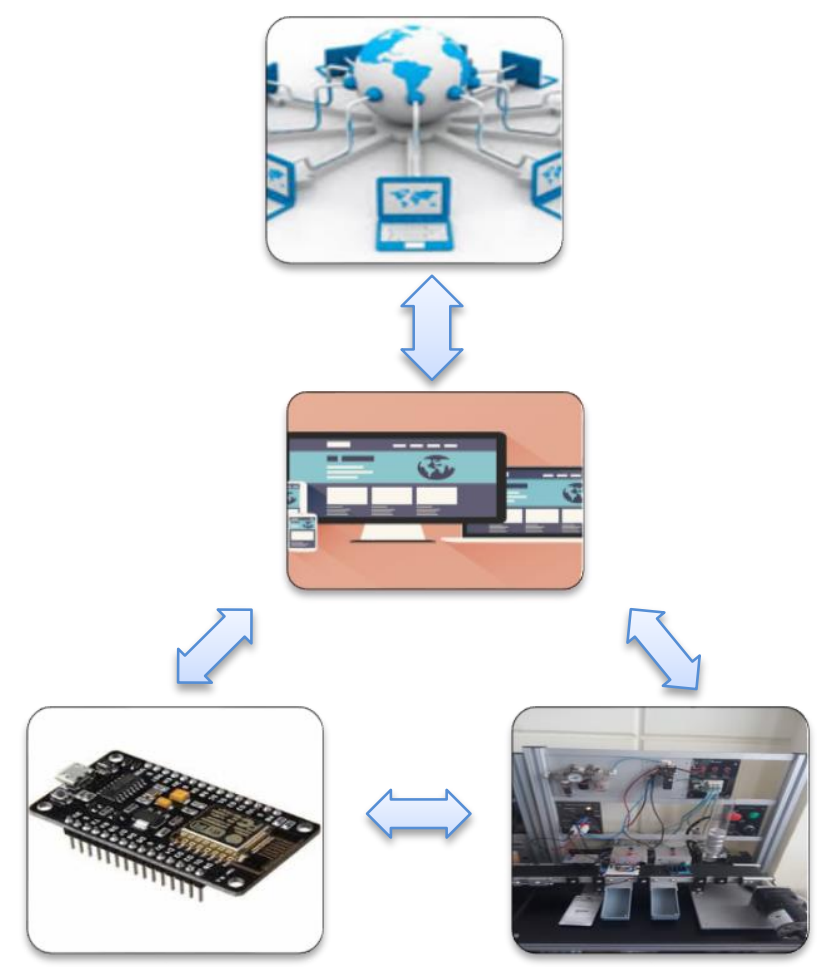

Figura 2 Modelo de comunicación entre PLC Siemens S7-200 y un sistema de IoT (nodemcu esp32 WIFI) Fuente: Elaboración Propia

\section{Diseño de interfaz de comunicación entre PLC siemens S7-200 y nodemcu esp32 WIFI}

La principal función de un PLC es la automatización de tareas, principalmente en procesos industriales donde el costo por desarrollo o manutención de sistemas automatizados es rentable (Jhosi 2016). La mayoría de los PLC modernos tienen la característica de comunicarse de una red a un sistema SCADA (Control de Supervisión y Adquisición de Datos) o bien en un navegador web.

De acuerdo con Bakshi (2019) las máquinas que controlan los procesos industriales trabajan con sistemas PLC-SCADA donde la adquisición de información es obtenida de sensores mediante señales digitales (I/O). Urrego (2012) establece que el uso de sensores industriales dentro de un proceso requiere de un tiempo de respuesta mayor cuando no se encuentran conectados a un sistema de comunicación.

Mohod (2016) considera el monitoreo y control industrial como una combinación de arquitecturas, mecanismos y algoritmos capaces de controlar los procesos industriales, motores, máquinas y diversos dispositivos para obtener un propósito.

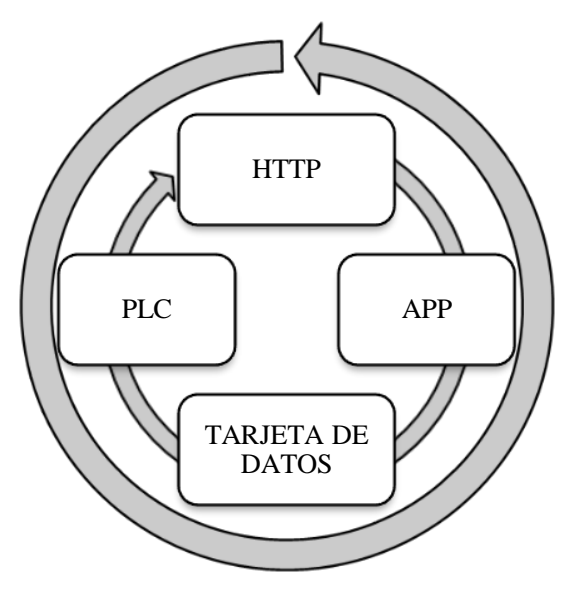

Figura 3 Arquitectura del sistema IoT-PLC Fuente: Elaboración Propia

La figura 3 muestra la arquitectura del sistema de comunicación IoT-PLC y la interfaz utilizada con el navegador web la cual tiene el control de los canales de entrada y salida, el vínculo entre el servidor web y el PLC es la tarjeta de adquisición de datos, mientras en la figura 4 se muestra el diagrama electrónico de acoplamiento de voltaje.

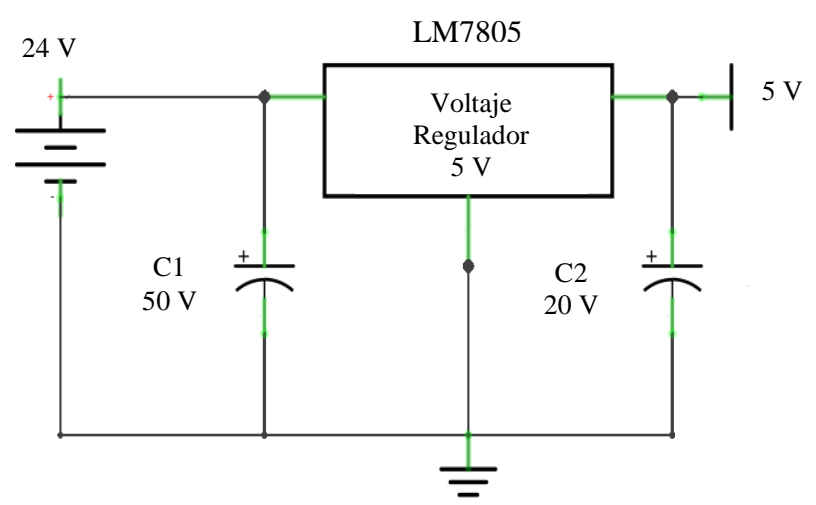

Figura 4 Diagrama del circuito regulador de voltaje de 24 a 5 volts

Fuente: Elaboración Propia en fritzing

\section{Sistemas de control industrial e IoT}

El control de procesos industriales mediante PLC y sistemas IoT se basan en un monitoreo continuo de sensores que recopilan los datos relevantes del proceso, esto determina el correcto funcionamiento del sistema (Rajesh 2017).

Uttam (2017) establece que conforme se utilice con mayor frecuencia el IoT en los procesos productivos, el sistema se vuelve más seguro por el monitoreo de datos en tiempo real, esto permite que el proceso aumente la estabilidad en el PLC. La figura 5 muestra el diagrama de control de un PLC. 


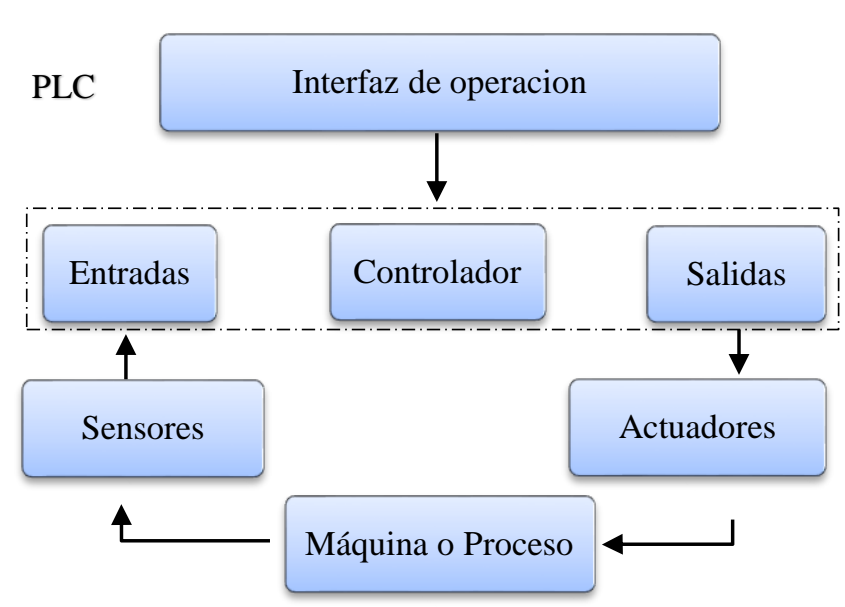

Figura 5 Diagrama de control de PLC

Fuente: Elaboración propia

\section{Implementación y construcción de comunicación}

El diagrama correspondiente al acoplamiento eléctrico para la conexión del sistema propuesto se muestra en la figura 6 , donde:

a. Es el regulador de voltaje con $24 \mathrm{VCD}$ de entrada correspondiente a la alimentación del PLC y 5 VCD de salida de la tarjeta de adquisición de datos.

b. El módulo Wifi correspondiente a la tarjeta de adquisición de datos, encargada de realizar la conexión de la red con el PLC.

c. El sistema de salida de control que permite manipular el PLC mediante una aplicación móvil o una página de internet.

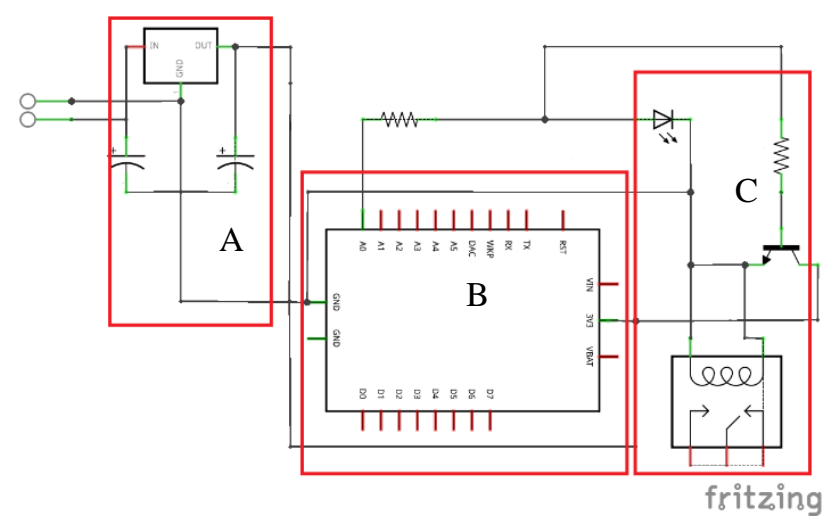

Figura 6 Diagrama sistema de comunicación wifi-PLC Fuente: Elaboración Propia

\section{Implementación y resultados}

Las características de diseño de un PLC permiten tener un campo de aplicación muy extenso, ya que, se utilizan principalmente en instalaciones que requieren de un proceso de maniobra, monitoreo, control y señalización.

Se utiliza un transportador industrial el cual identifica materiales por medio de un sensor capacitivo y otro inductivo. Los elementos del transportador industrial se muestran en la figura 7.

1. Sistema neumático para realizar accionamiento de pistones

2. Sistema de control diseñado bajo la tarjeta nodemcu esp32 WIFI

3. Sistema de electroválvulas a 24 VDC

\section{Motor trifásico}

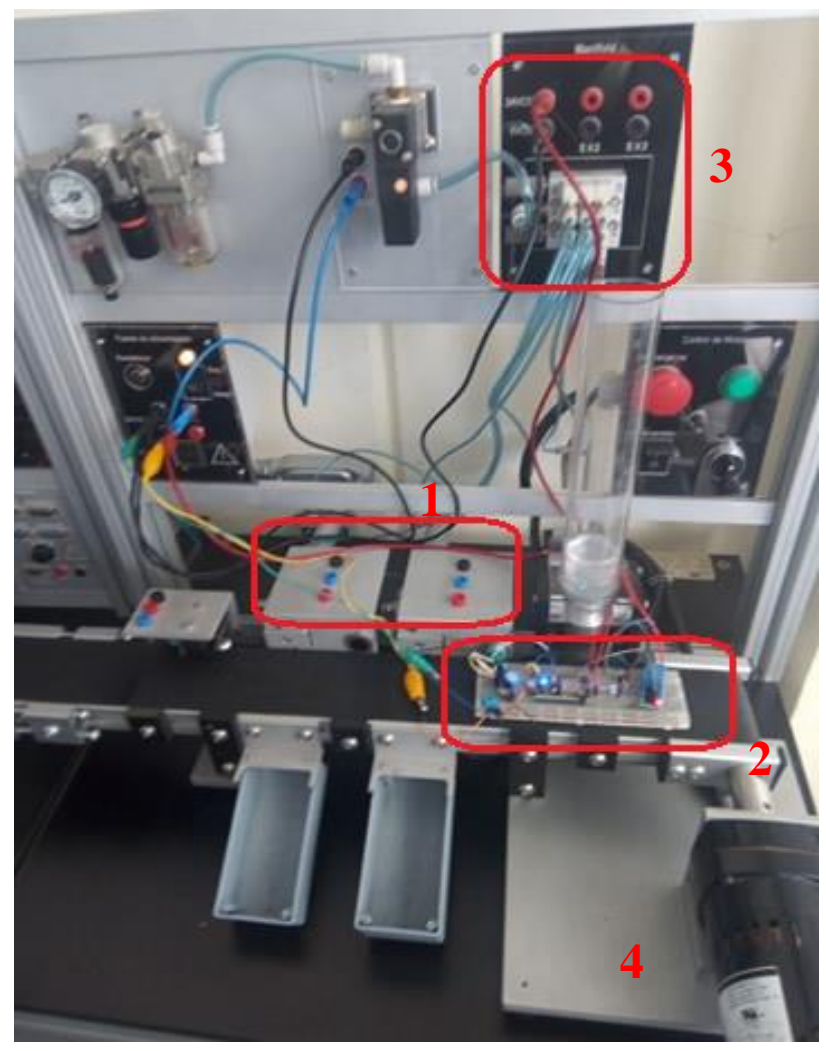

Figura 7 Sistema de comunicación wifi-PLC Fuente: Elaboración Propia 


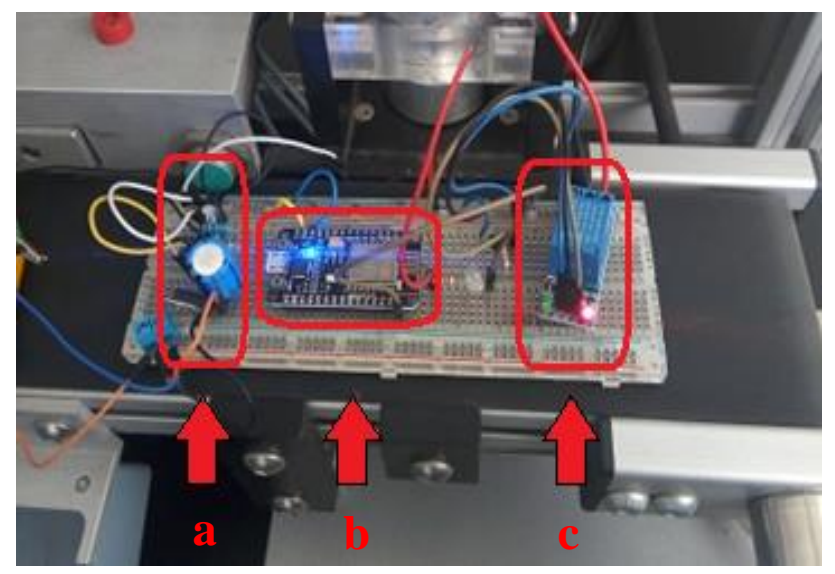

Figura 8 Acoplamiento eléctrico de comunicación WifiPLC

Fuente: Elaboración Propia

La figura 8 muestra el acoplamiento eléctrico descrito en la figura 6.
a. Regulador de voltaje
b. Tarjeta de comunicación
c. Sistema de control al PLC

Para validar el correcto funcionamiento del dispositivo se evalúa en su etapa de control (accionamiento de las electroválvulas) a través de un dispositivo móvil, la respuesta de salida obtenida al cargar y descargar el sistema de comunicación se observa en la figura 9 .

La figura 9 contiene la respuesta del accionamiento de las electroválvulas de señal de salida el cual se comporta como la carga y descarga de una inductancia

1. Acción de carga

2. Acción de descarga

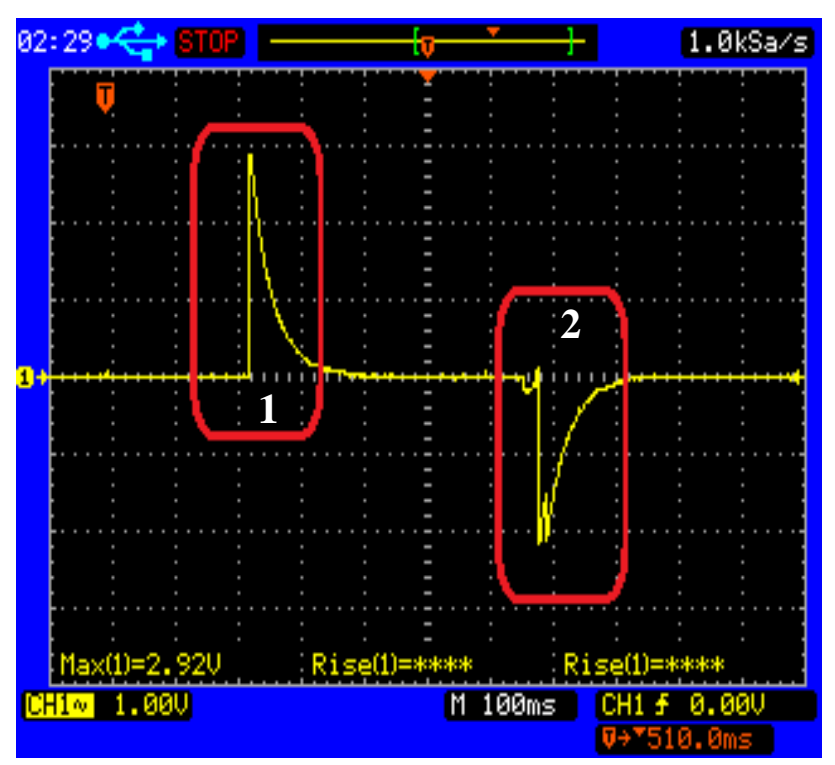

Figura 9 Respuesta de salida entre sistema Wifi y PLC Fuente: Elaboración Propia
Como puede verse en el grafico 9 la respuesta de salida para el control del sistema de comunicación está basada en impulsos donde solamente manda la cantidad de energía necesaria y posteriormente el sistema regresa a su normalidad.

\section{Agradecimiento}

Los autores agradecen a la Universidad Autónoma del Estado de México a través del programa PFCE-2019 asignado a la UAP Tianguistenco.

\section{Conclusiones}

El aporte de diversas tecnologías involucradas en la Industria 4.0 tiene como principio el modificar los procesos de fabricación de las empresas, de tal manera que generará una mayor productividad al tener un sistema de comunicación máquina a máquina supervisado y controlado para la toma de decisiones.

En este trabajo se muestra que el acoplamiento entre un PLC comercial y un microcontrolador basado en IoT se consigue una comunicación segura, confiable y robusta, abriendo nuevas posibilidades de control remoto.

\section{Trabajos futuros}

Los avances referentes a la Industria 4.0 van incrementando de una manera rápida, siendo el Internet de las Cosas Industrial una de las tecnologías más importantes, donde su principal aportación se basa en la comunicación del Internet con dispositivos propios de la industria, esto deja una cantidad de oportunidades para desarrollar trabajos futuros encaminados a:

- Mejorar el sistema de conexión a internet por medio de Ethernet y otros medios de comunicación móviles.

- Desarrollar un sistema un sistema seguro ante amenazas cibernéticas.

- Utilizar la ventaja computacional que tiene los microcontroladores sobre los PLC comerciales, para desarrollar controles robustos basados en redes neuronales realizando una comparación con respecto a los controles clásicos PID. 


\section{Referencias}

Ahmed, N., De, D., and Hussain, M. I. (2018). "Internet of Things (IoT) for Smart Precision Agriculture and Farming in Rural Areas". IEEE Internet of Things Journal, 1-1. DOI:10.1109/JIOT.2018.2879579.

Bakshi, S., Khairmode, G., Varkhede, N. amd Ayane, S. (2019). "Monitoring and control of PLC based automation system parameters using IoT" Industrial Automation using IoT". International Research Journal of Engineering and Technology (IRJET), Vol 6, ISSN 23950056.

Cheng, X., Zhang, R., \& Yang, L. (2018). "Wireless Towards the Era of Intelligent Vehicles". IEEE Internet of Things Journal, 1-1. DOI:10.1109/JIOT.2018.2884200.

Esfahani, A., Mantas, G., Matischek, R., Saghezchi, F. B., Rodriguez, J., Bicaku, A., Bastos, J. (2017). "A Lightweight Authentication Mechanism for M2M Communications in Industrial IoT Environment". IEEE Internet of Things Journal, 1-1. DOI:10.1109/JIOT.2017.2737630.

Humayed, A., Lin, J., Li, F., and Luo, B. (2017). "Cyber-Physical Systems Security-A Survey". IEEE Internet of Things Journal, 4(6), 18021831. DOI:10.1109/JIOT.2017.2703172.

Jhosi, R., Javad, H. M., Mali, A. and Kulkani, S. V. (2016). "IOT Application for Real-time Monitoring of PLC Data using EPICS". International Conference on Internet of Things and Applications (IOTA). Pune India 2016.

Karthika, R.A., Rahamtula, S., and Anusha, Y. (2018) "Internet of things for industrial monitoring and control applications". International Journal of Engineering and Techonology, Vol. 7, 280-282.

Lau, B. P. L., Wijerathne, N., Ng, B. K. K., \& Yuen, C. (2018). "Sensor Fusion for Public Space Utilization Monitoring in a Smart City". IEEE Internet of Things Journal, 5(2), 473-481. DOI:10.1109/JIOT.2017.2748987.
Minoli, D., Sohraby, K., and Occhiogrosso, B. (2017). "IoT Considerations, Requirements, and Architectures for Smart Buildings Energy Optimization and Next Generation Building Management Systems". IEEE Internet of Things Journal, 1-1. DOI:10.1109/JIOT.2017.2647881. Mohod, S., and Deshmukh, R. (2016). "Internet of Things for Industrial Monitoring and Control Applications". International Journal of Scientific \& Engineering Research, Vol. 7, ISSN 22295518 .

Muñiz, R. (2019). "Diseño y construcción de un Sistema de inmersión temporal de bajo costo para la propagación in vitro de plantas bajo el enfoque de una tecnología apropiable". Tekhné. 22(2). ISSN 1316-3930.

Ngu, A. H. H., Gutierrez, M., Metsis, V., Nepal, S., and Sheng, M. Z. (2016). "IoT Middleware: A Survey on Issues and Enabling technologies". IEEE Internet of Things Journal, 1-1. DOI:10.1109/JIOT.2016.2615180.

Pancha, J., Rojas, V., Lema, J. and Arteaga, G. (2019). "Mathematical Statistical Analysis About the behavior of the Electrical Conditions Established under the ISO Standard Test 1675022 in Two Automotive Alarm Modules in Ecuador”. Revista Politécnica, 42(2), 49-56.

Pinto, V., and Arias, O. (2019). "Módulo de control de potencia suministrada por un generador a la red eléctrica". Universidad del Norte. Colombia 2019.

Quispe, A. and Maquera, J.C. (2019) "Diseño y construcción de un módulo de laboratorio para el análisis de los parámetros de una bomba centrifuga de 1 HP como turbina". Universidad Nacional del Altiplano, Perú 2019.

Qian, Y., Yan, J., Guan, H., Li, J., Zhou, X., Guo, S., and Jayakody, D. N. K. (2017). "Design of Hybrid Wireless and Power Line Sensor Networks with Dual-Interface Relay in IoT". IEEE Internet of Things Journal, 1-1. DOI:10.1109/JIOT.2017.2725451.

Rajesh, G., Renuka, S., Parthipan, V. and Mizpah, J. (2017). "Industrial machine control and monitoring system using Io $T$ ". International Journal of Pure and Applied Mathematics. Vol. 116. 105-111. 
Schwab, K. (2016). "La cuarta revolución industrial". World Economic Forum. El tiempo casa editorial S.A. ISBN: 978-84-9992-699-5.

Tietz, F., Brandao, D., and Alves, L. F. (2018). "Development of an Internet of Things Gateway Applied to a Multitask Industrial Plant". (2018) 13th IEEE International Conference on Industry Applications (INDUSCON).

DOI:10.1109/induscon.2018.8627273.

Urrego, D. A. (2012). "Desarrollo de sistema Potable para la Medición del Gasto Energético". Universidad de Antioquia, Medellín Colombia 2012.

Uttam, B., Baspusahed, G., Shivaji, J. and Pisal, R. (2017) "Industrial Automation using IoT". International Research Journal of Engineering and Technology (IRJET), Vol 4, ISSN 23950056.

Zhang, Q., Liu, M., Lin, X., Liu, Q., Wu, J., and Xia, P. (2018). "Optimal Resonant Beam Charging for Electronic Vehicles in Internet of Intelligent Vehicles". IEEE Internet of Things Journal, 1-1. DOI:10.1109/JIOT.2018.2872431. 